\title{
ARTICLE OPEN Continuous strengthening in nanotwinned diamond
}

\author{
Bin Wen ${ }^{1,3}$, Bo Xu $\mathbb{D}^{1,3}$, Yanbin Wang $\mathbb{D}^{2}$, Guoying Gao ${ }^{1}$, Xiang-Feng Zhou ${ }^{1}$, Zhisheng Zhao ${ }^{1}$ and Yongjun Tian ${ }^{1 *}$
}

Strengths of nanograined (ng) and nanotwinned (nt) metals increase with decreasing grain size and twin thickness, respectively, until reaching a critical value, below which strength decreases. This behavior is known as the reverse Hall-Petch effect (RHPE), which has also been observed in nanograined cubic boron nitride (cBN) and diamond. Surprisingly, however, hardness of nt-cBN and nt-diamond increases continuously with decreasing twin thickness down to several nanometers, suggesting the absence of RHPE in these covalent materials. The mechanism responsible for such a behavior remains controversial. Here we investigate the strengthening mechanisms in ng- and nt-diamond using molecular dynamics and first-principles calculations. For ng-diamond, the competition between shuffle-set dislocation (SSD) and grain boundary atom motions gives rise to RHPE. For nt-diamond, SSDs remain dominant but their slips along twin boundaries energetically show no advantage over those along other slip planes. Twin domains are locked and mechanically stable, resisting SSD propagation and inhibiting RHPE. These findings provide new insights into the hardening mechanism of nanotwinned covalent materials.

npj Computational Materials (2019)5:117; https://doi.org/10.1038/s41524-019-0256-2

\section{INTRODUCTION}

Nanostructuring can introduce immense amounts of interfaces such as grain boundaries (GBs) and twin boundaries (TBs) into a material, reducing dislocation pile-ups near the boundaries, thereby hindering dislocation transmission and increasing resistance to deformation. ${ }^{1}$ This dislocation-blocking effect becomes more significant with decreasing grain size $(d)$ and twin thickness $(\lambda)$, leading to strengthening of materials known as the Hall-Petch effect. ${ }^{2,3}$ For metals, however, the dislocation-blocking effect diminishes when $d$ or $\lambda$ reaches a critical value, ${ }^{4,5}$ below which GB sliding (GBS) or detwinning becomes dominant. Further reducing $d(\lambda)$ would induce more GBS (detwinning), resulting in softening of nanocrystalline metals, ${ }^{6-12}$ a mechanism known as the reverse Hall-Petch effect (RHPE).

RHPE also occurs similarly in nanograined (ng) covalent materials such as cubic boron nitride (cBN) and diamond, ${ }^{13,14}$ where hardness is used as the indicator for strength. In nanotwinned (nt) diamond and $\mathrm{CBN}$, however, hardness continuously increases with decreasing twin thickness to below $5 \mathrm{~nm},{ }^{15,16}$ contrasting strikingly to that of nt-metals. The mechanism responsible for the continuous hardening in nt-diamond and nt-cBN is currently a hotly debated subject. ${ }^{17,18}$ In this study, we examine various deformation modes under uniaxial compressive stress in ng- and nt-diamond using molecular dynamics (MD) simulations and first-principles calculations, with the aim to clarify the corresponding plastic deformation mechanisms. Our results indicate that, in nt-diamond, shuffle-set dislocations (SSDs) dominate the plastic deformation but their slips along TBs do not have advantage energetically over those along other slip planes. TBs are mechanically stable and resist SSDs propagation across twin domains, leading to continuous operation of the Hall-Petch effect at the nanoscale level. This is in stark contrast to nt-metals, where detwinning becomes increasingly active as $\lambda$ is reduced to below ca. $15 \mathrm{~nm}$, due to collective slips of partial dislocations parallel to TBs. ${ }^{6,9,10}$

\section{RESULTS}

MD Simulations and First-Principles Calculations

We performed MD simulations on a variety of three-dimensional (3D) ng-diamond and nt-diamond samples with average grain sizes ranging from 2 to $25 \mathrm{~nm}$ (see Methods summary). Supplementary Figure 1 shows representative ng-diamond and nt-diamond samples under investigation. The samples were compressed with a constant strain rate of $5 \times 10^{8} \mathrm{~s}^{-1}$ (i.e., a displacement-controlled compression process) with Tersoff poten$\mathrm{tial}^{19}$ and the corresponding uniaxial compressive stress-strain curves were calculated, as shown in Supplementary Figs. 2 and 3. The stress-strain curves can clearly be divided into elastic and plastic deformation regimes. In the elastic deformation regime, the stress increases almost linearly with increasing strain to a peak value. After that, the plastic deformation occurs and the stress decreases rapidly to a stabilized value. It is noteworthy that there is no obvious work hardening observed, indicating the dislocation density is relatively low during plastic deformation. Setting the peak stress of the stress-strain curve as the corresponding compressive strength, we can plot the compressive strength of ng-diamond and nt-diamond as functions of $d$ and $\lambda$, respectively, as shown in Fig. 1. For ng-diamond (Fig. 1a), compressive strength increases with decreasing $d$, reaching a maximum of $153 \mathrm{GPa}$ at $d \approx 8 \mathrm{~nm}$, below which strength decreases with further decreasing $d$. For nt-diamond (Fig. 1b), compressive strength shows strong dependence on both $d$ and $\lambda$, and is consistently greater than that of ng-diamond with the same $d$. For a given $d$, strength of nt-diamond increases continuously with decreasing $\lambda$ down to the minimum value of $0.618 \mathrm{~nm}\left(=3 d_{111}\right.$, i.e., one unit-cell length along $<111>$ direction). As suggested by the Tabor relation, ${ }^{20}$ the indentation hardness is proportional to tensile yield strength for metals. Same as in metals, the plastic deformation in diamond is also dislocation controlled. The uniaxial compressive strength therefore can be used as the indicator of hardness for diamond. This continuous strengthening revealed for nt-diamond is in sharp contrast to what occurs in ng-diamond as well as ng- and ntmetals, but consistent with the experimental observations of

${ }^{1}$ Center for High Pressure Science, State Key Laboratory of Metastable Materials Science and Technology, Yanshan University, Qinhuangdao 66004, China. ${ }^{2}$ Center for Advanced Radiation Sources, University of Chicago, Chicago, IL 60439, USA. ${ }^{3}$ These authors contributed equally: Bin Wen, Bo Xu. *email: fhcl@ysu.edu.cn 

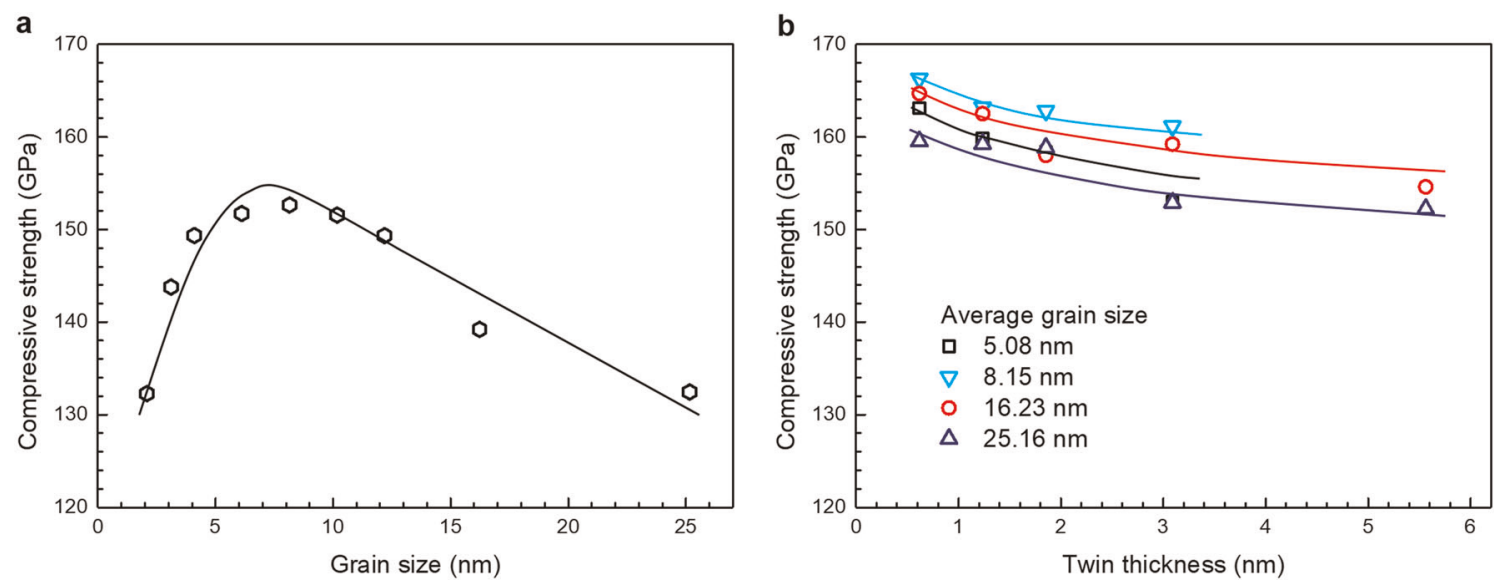

Fig. 1 Compressive strength as a function of grain size and twin thickness for $\mathrm{ng}$-diamond and $\mathrm{nt}$-diamond samples at $300 \mathrm{~K}$ under displacement-controlled compression with Tersoff potential. a Compressive strength of ng-diamond as a function of $d$. Maximum compressive strength is achieved with an average $d$ of ca. $8 \mathrm{~nm}$. b Compressive strength of nt-diamond as a function of $\lambda$. The continuously increasing strength with decreasing $\lambda$ down to the minimum value $0.618 \mathrm{~nm}$ is noteworthy. The lines are guide to the eyes.

hardening in nt-diamond. ${ }^{16}$ To further corroborate this result, displacement-controlled compression process with Albe analytical potential $^{21}$ and force-controlled compression process ${ }^{22,23}$ with Tersoff potential have been performed. As shown in Supplementary Figs. 4-10, similar variation trends of compressive strength as functions of $d$ and $\lambda$ are revealed for ng-diamond and nt-diamond, respectively, thus verifying the reliability of our result.

\section{DISCUSSION}

To clarify the different behaviors in ng- and nt-diamond, we investigated deformation modes in five representative samples, i.e., two ng-diamonds with average grain sizes of $3.11 \mathrm{~nm}$ and $16.23 \mathrm{~nm}$, two nt-diamonds with an identical average $d$ of $16.23 \mathrm{~nm}$ but different twin thicknesses of $0.618 \mathrm{~nm}$ and $5.562 \mathrm{~nm}$, and one nt-diamond with average $d$ of $5.08 \mathrm{~nm}$ and $\lambda$ of 0.618. A displacement-controlled compressive stress with a constant strain rate of $5 \times 10^{8} \mathrm{~s}^{-1}$ was applied along one direction (see the arrows in Supplementary Fig. 1), leaving the perpendicular directions free. Distinct deformation modes were identified based on relative displacements of atoms with respect to the undeformed sample (Fig. 2 and Supplementary Figs. 11 and 12) and dislocation activities (Supplementary Videos 1-5).

For ng-diamond with smaller grain size $(3.11 \mathrm{~nm}$, Supplementary Fig. 11a-c, and Supplementary Video 1), a few dislocations are distributed along GBs with no free dislocations in grain interiors. With increasing true strain from 0 to $30 \%$, relative displacements of atoms occur increasingly near GBs, consistent with previous simulation study on metals. ${ }^{7}$ No dislocations slip across grain interiors during the compressing process. For ng-diamond samples with larger grain size $(16.23 \mathrm{~nm}$, Supplementary Fig. $11 \mathrm{~d}-\mathrm{f}$, and Supplementary Video 2), relative displacements of atoms increase primarily around GBs with true strains up to $13 \%$. It is noteworthy that the percentage of GB atoms in the larger grained ng-diamond sample $(9.9 \%)$ is significantly lower than that in the smaller grained one (45.7\%). Therefore, the total contribution of GB atoms to plastic deformation is significantly less in the sample with $d=16.23 \mathrm{~nm}$. Further deformation (true strain $>13 \%$ ) introduces SSDs traversing the grains and SSD slip becomes the dominant deformation mode. The GB atoms mediated deformation and SSDs mediated one compete along with varying grain size. A transition from GB-atom-dominated to SSD-dominated mechanism occurs at $d$ of ca. $8 \mathrm{~nm}$, where the strongest compressive strength is achieved (Fig. 1a). The turnover of compressive strength (i.e., RHPE) is similar to that observed in ng fcc metals. 7,24
For nt-diamonds with $d=16.23 \mathrm{~nm}$, both grain interiors and TBs remain dislocation free with true strains up to $17 \%$ and $15 \%$ for samples with $\lambda$ of 0.618 and $5.562 \mathrm{~nm}$, respectively (Supplementary Videos 3 and 4). All dislocations are located at GBs and the deformation is dominated by GB-atom-mediated motion, with no contribution from TBs. Above the aforementioned strain levels, deformation is primarily controlled by slips of SSDs that cut across TBs and traverse grain interiors (Fig. 2 and Supplementary Fig. 12a-c). TBs hinder dislocation slips and resist deformation. Smaller $\lambda$ corresponds to higher density of TBs, where dislocation motions are blocked more effectively. As a result, compressive strength increases monotonically with decreasing $\lambda$ (Fig. 1b). For ntdiamond with $d=5.08 \mathrm{~nm}$ and $\lambda=0.618 \mathrm{~nm}$, with increasing true strain from 0 to $30 \%$, relative displacements of atoms occur increasingly near GBs during the compressing process with no dislocations slipping across grain interiors (Supplementary Fig. $12 \mathrm{~d}-\mathrm{f}$ and Supplementary Video 5), similar to what occurs in ng-diamond with $d=3.11 \mathrm{~nm}$.

The continuous strengthening/hardening of nt-diamond with decreasing $\lambda$ is fundamentally different from that of nt metals. In nt-Cu, e.g., plastic deformation at room temperature is dominated by $\frac{1}{6}<112>$ partial dislocations slipping parallel to TBs when $\lambda$ is smaller than a critical value of ca. $15 \mathrm{~nm},{ }^{10}$ leading to detwinning and reduced strength. ${ }^{6}$ To elucidate the contrasting deformation mechanisms in nt-diamond and nt metals, generalized stacking fault energy (GSFE) curves are evaluated with first-principles calculations for diamond, cBN, Al, Cu, and Ni (Fig. 3, Supplementary Figs. 13-15, and Supplementary Table 1).

The ratios of intrinsic stacking fault energy $\left(\gamma_{\text {isf }}\right)$ to unstable stacking fault energy $\left(\gamma_{\text {usf }}\right)$ for $\mathrm{Al}, \mathrm{Cu}$, and $\mathrm{Ni}$ vary in a wide range (Supplementary Table 1). For each fcc metal examined here, $\gamma_{\text {usf }}$ $\gamma_{\text {utf }}$ (unstable twin formation energy) and $\gamma_{\text {utm }}$ (unstable twin migration energy) are clearly distinguishable (Supplementary Fig. 13). It is a common feature for metals that $\gamma_{\mathrm{utm}}$ is generally lower than

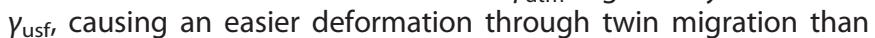
through the formation of stacking faults from Shockley partial dislocations. As a result, collective glide of multiple Shockley partial dislocations induces detwinning, ${ }^{6}$ which becomes increasingly dominant when twin thickness is less than a critical value $\lambda_{c^{\prime}}$ ca. $15 \mathrm{~nm}$ for $\mathrm{nt}-\mathrm{Cu}^{25}$ (Supplementary Fig. 16a). Such a process would further decrease critical resolved shear stress (CRSS) for partial dislocations slipping parallel to TBs with decreasing $\lambda$, providing a mechanistic explanation of RHPE observed in $\mathrm{nt}-\mathrm{Cu}^{10}$ : Plastic deformation is dominated by collective glide of multiple Shockley partial dislocations when $\lambda$ is lower than a critical value of $15 \mathrm{~nm}$, resulting in a turnover of strength or hardness. 


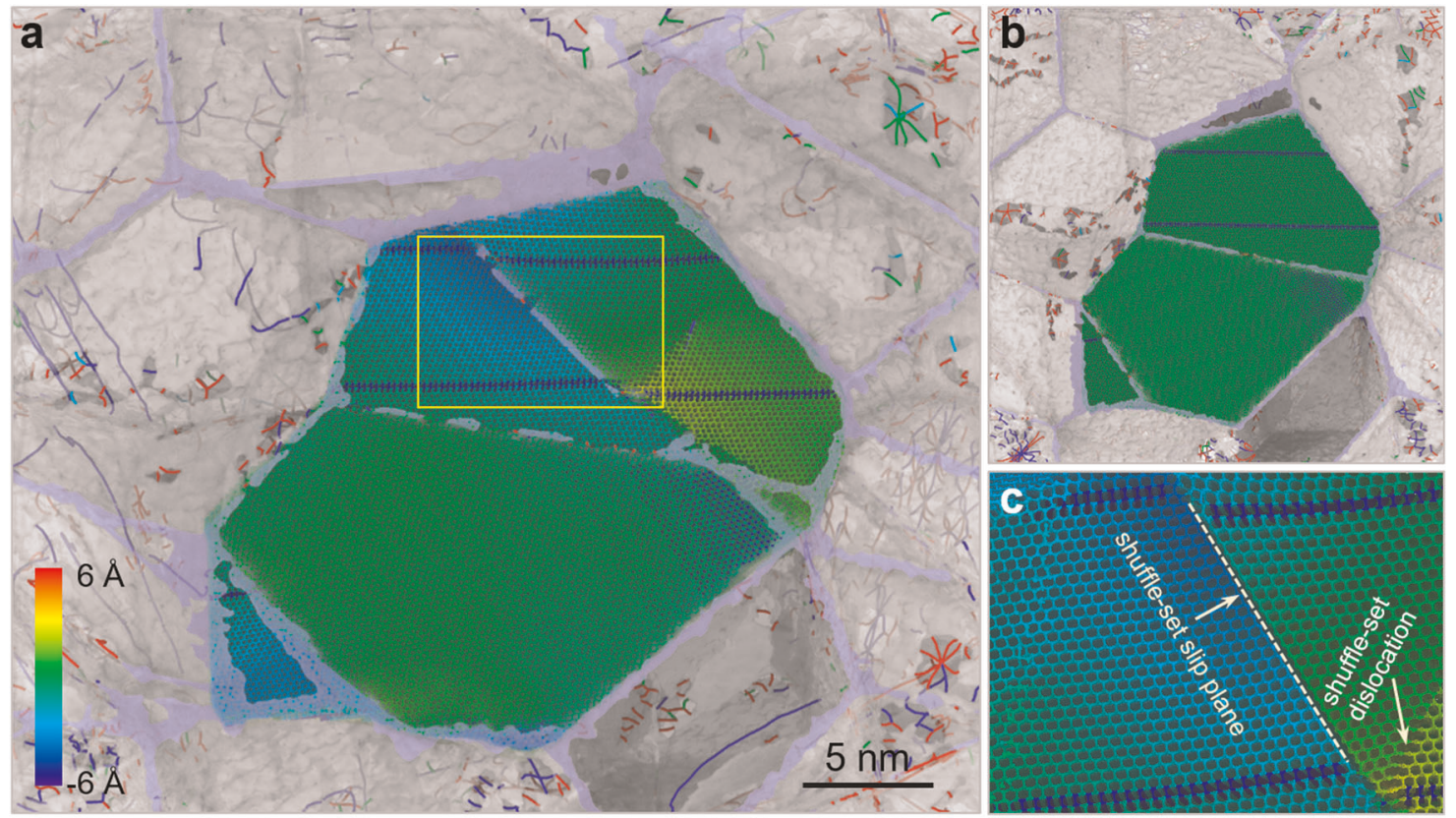

Fig. 2 Deformation modes simulated with Tersoff potential in nt-diamond with an average grain size of $16.23 \mathrm{~nm}$ and an average twin thickness of $\mathbf{5 . 5 6 2} \mathbf{~ n m}$. a A cross-section snapshot of the nt-diamond sample under a true compressive strain of $15.75 \%$. Atoms are colored by their relative displacements with respect to the undeformed states. Blue-colored atoms are located near the twin boundaries, cyan-colored regions indicate grain boundaries, blue and red lines indicate shuffle-set dislocations, green lines $\frac{1}{6}<112>$ glide-set Shockley partial dislocations. For simplicity, atoms are made resolved for the central grains, while only defects are shown for other grains. b The original undeformed nt-diamond sample. c Enlarged image for the yellow rectangle in a, emphasizing the detail of a nanotwin with a shuffle-set dislocation cutting across.

In contrast to metals, $\gamma_{\text {usf, }} \gamma_{\text {utf }}$, and $\gamma_{\text {utm }}$ of diamond are virtually identical (Supplementary Fig. 14). Diamond consists of two interpenetrating face-centered cubic sublattices that shift relatively by one-fourth the body diagonal of the unit cell, leading to two nonequivalent $\{111\}$ slip planes: glide-set (within the densely populated compound layers, Fig. 3a) and shuffle-set (between adjacent compound layers, Fig. 3b). Moreover, carbon atoms form strong and highly directional covalent bonds, which are fundamentally different from those in fcc metals (Supplementary Fig. 17). Such structural and bonding features profoundly influence active slip modes in diamond. An ideal slip along the glide-set plane involves three $\mathrm{C}-\mathrm{C}$ bonds per atom (either severing two $\mathrm{C}-\mathrm{C}$ bonds with the third one compressed along the [110] direction or severing one $\mathrm{C}-\mathrm{C}$ bond with the other two compressed along $[11 \overline{2}])$, whereas slip along the shuffle-set plane only involves one $\mathrm{C}-\mathrm{C}$ bond per atom (Fig. 3a, b). Therefore, the force needed to start a glide-set slip is roughly triple that for a shuffle-set slip, which is further confirmed in the calculated restoring stresses (Fig. 3d). It is worth noting that the plastic deformation in diamond is mostly force-activated at low temperature. The thermal activation is highly restrained due to high barrier for dislocation motion, stemming from the strong and highly directional covalent bonds. Even though $\gamma_{\text {usf }}$ along the shuffle-set [1 $\left.1 \overline{1} 0\right]$ and [11 $]$ directions is higher than that along glide-set $[11 \overline{2}]$ direction (Fig. $3 c$ ), the maximal restoring stresses for these cases are similar, with a much smaller slope for the shuffle-set slips (Fig. 3d). In addition, slips of glide-set Shockley partial dislocations are energetically more costly due to the extra stacking fault energy involved. All these factors make slip of SSDs the favored plastic deformation mode. This agrees well with our MD simulations for ng- or nt-diamond, where deformation is dominated by SSDs cutting across GBs and TBs, with less twinning or detwinning by $\frac{1}{6}<112>$ glide-set partial dislocations (density of $\frac{1}{6}<112>$ glide-set partial dislocation is only $30 \%$ that of SSDs). Notably, the stacking fault energies do not vary significantly with distance away from twin planes (Supplementary
Fig. 15), implying that SSDs are essentially not affected by TBs in nt-diamond.

Knowledge on the CRSS for detwinning and Shockley partial dislocation sliding parallel to TBs allows us to estimate the critical twin thickness, $\lambda_{c}$, for both metals and diamond (Supplementary Fig. 16). Due to the high lattice friction stress of diamond, ${ }^{26}$ a very low $\lambda_{c}$ of ca. $0.35 \mathrm{~nm}$ is deduced, which is significantly lower than that of nt-Cu ${ }^{27}$ and even lower than the minimum twin thickness of diamond. Therefore, detwinning process cannot be activated in diamond. As a result, RHPE is absent with decreasing $\lambda$, leading to continuous strengthening to the minimum twin thickness. The superior stability of TB over GB in diamond are further confirmed by comparing the temperature-dependent compressive strengths of diamond with different grain size or twin thickness. As shown in Supplementary Fig. 18, the compressive strength for ng-diamond with different grain size reverses at a critical temperature. No such reversal occurs in nt-diamond with different twin thickness for temperature as high as $1900 \mathrm{~K}$, corroborating the aforementioned result. ${ }^{28}$

Figure 4 schematically illustrates the strength of nanostructured copper and diamond as functions of grain size or twin thickness. For ng metals and diamond, even though GBs resist dislocation transmission and contribute to strengthening, CRSS along GBs is inevitably lower than that along perfect crystal slip planes. The competition between dislocation glide and GBS gives rise to an optimal grain size $d_{c}$, with which the material is strongest. ${ }^{24}$ Above $d_{c}$ dislocation glide becomes the primary deformation mode and materials strengthen with further decreasing grain size, and this is the Hall-Petch effect. Below $d_{c}$, GBS becomes the primary deformation mode and materials soften with further decreasing grain size, ${ }^{23}$ and this is RHPE. For nt-diamond and -metals, the Hall-Petch effect is in operation with large $\lambda$ where the dislocation cut across TBs is the primary deformation mode. However, the parallel in mechanical response ends with further decreasing $\lambda$. For nt-metals such as $\mathrm{Al}, \mathrm{Cu}$, and $\mathrm{Ni}$, the slips of Shockley partial 
a
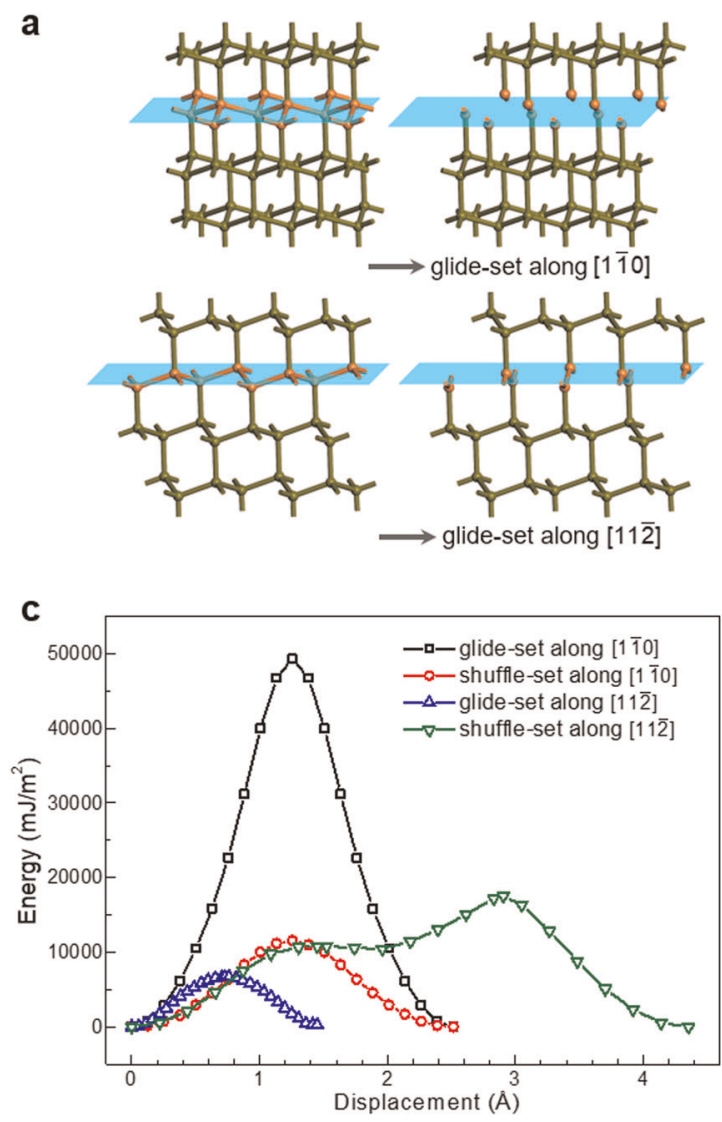

b
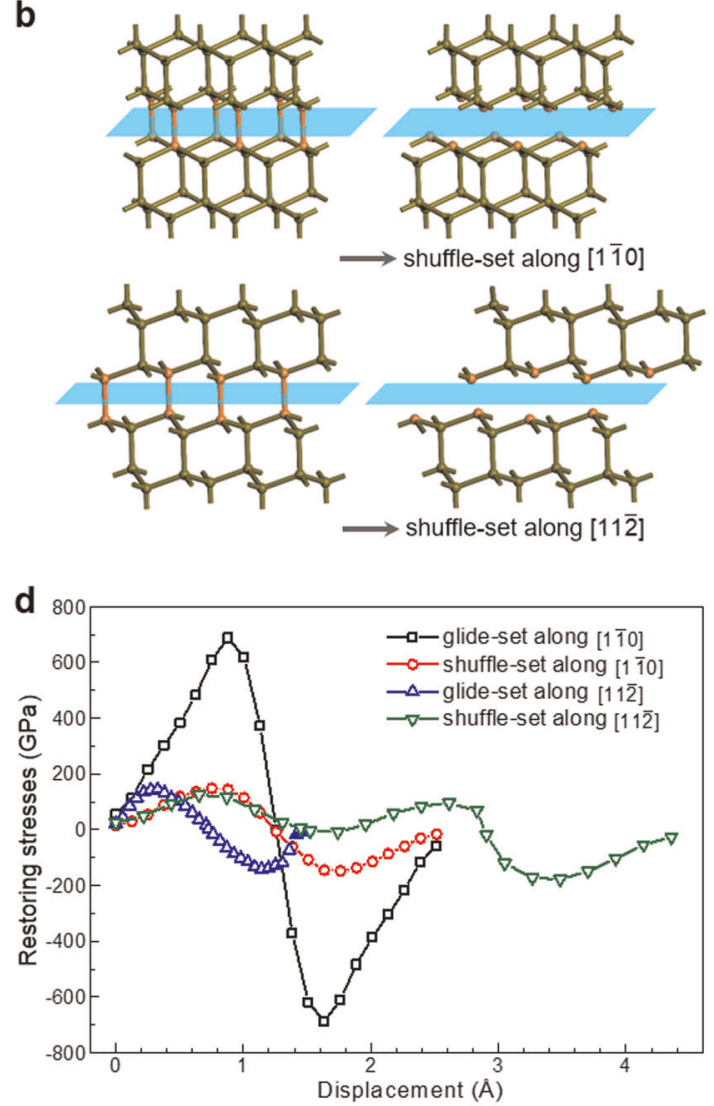

Fig. 3 Stacking fault energies and restoring stresses along different slip planes and slip directions in diamond. a Atomic configurations of the initial/final and intermediate states for glide-set along [1 $1 \overline{1} 0]$ and $[11 \overline{2}$ directions, respectively. $\mathbf{b}$ Atomic configurations of the initial/final and intermediate states for shuffle-set along [110] and [112] directions, respectively. Atoms and chemical bonds that undergo severe deformation are colored in dark orange. The cyan cross-sections indicate the slip planes; arrows indicate the slip directions. c GSFE curves for four different slip modes in diamond, due to two distinct slip planes, glide-set and shuffle-set, and two slip directions, [1 $\overline{1} 0]$ and [112]. d Restoring stresses, derivatives of stacking fault energies with respect to slip distance.

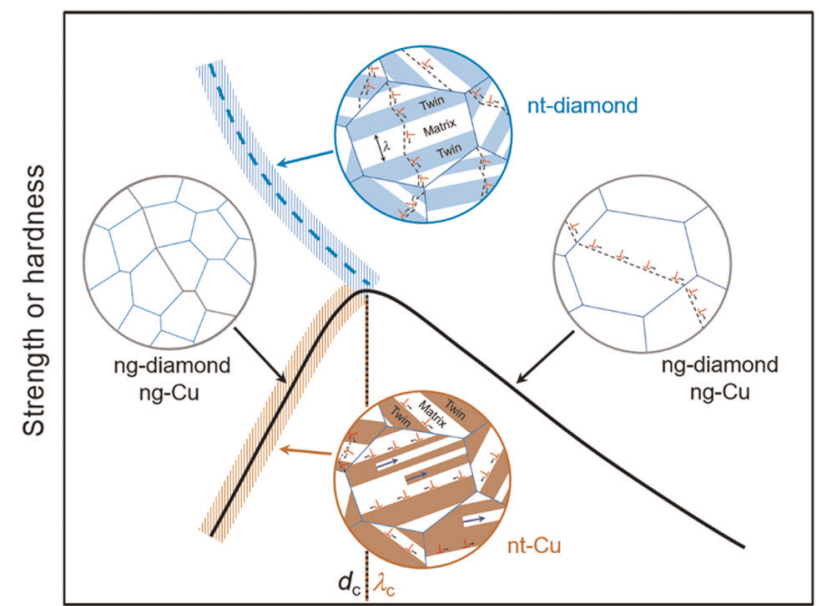

Grain size or twin thickness

Fig. 4 Diagram of ng-diamond and ng-Cu (nt-diamond and nt-Cu) strength as a function of grain size (twin thickness). The black line summarizes the main feature of $\mathrm{ng}$-diamond and $\mathrm{ng}-\mathrm{Cu}$ where a turn from the Hall-Petch effect to RHPE occurs at $d=d_{c}$; the brown patterned area indicates RHPE in nt-Cu with $\lambda<\lambda_{c}$ (the Hall-Petch effect dominates with $\lambda>\lambda_{c}$ ); the cyan dash line and patterned area emphasize the continuous strengthening of nt-diamond with decreasing $\lambda$. dislocations parallel to TBs are much easier near TBs than far from TBs, leading to detwinning that become dominant with $\lambda$ below $\lambda_{c}$ (which is a critical twin thickness from Hall-Petch effect to RHPE), as indicated by the blue arrows in Fig. 4. This process contributes to RHPE, which is ubiquitously observed in nt-metals. ${ }^{5,9,10}$ For ntdiamond, energy requirements for SSDs slipping along TBs and other slip planes far away from TBs are virtually identical, and $\lambda_{c}$ for activating detwinning process is extremely small (ca. $0.35 \mathrm{~nm}$ ). This means that the critical twin thickness $\lambda_{c}$ from Hall-Petch effect to RHPE for nt-diamond is less than a unit-cell length along the $<111>$ direction and it can explain the continuous hardening in nt-diamond and nt-cBN where $\lambda$ is $<5 \mathrm{~nm}$ with $d$ in the range of $20-50 \mathrm{~nm}$ and $30-150 \mathrm{~nm}$, respectively. ${ }^{15,16}$

Our simulations evidence the Hall-Petch effect is continuously operative in nt-diamond with $\lambda$ down to the lowest value. This provides an alternative dimension for tailoring properties and performance of materials with "hard" twin planes (usually covalent materials). For these materials, a practical strategy to achieve super-strength and/or superhardness is to introduce more TBs into the microstructure.

\section{METHODS}

\section{Simulation}

To build 3D ng-diamond samples containing randomly orientated grains, a periodic cube was segmented into 3D Voronoi convex polyhedrons, ${ }^{29}$ with 
each convex polyhedron filled with randomly orientated diamond grains. It is noteworthy that atoms at the GBs, which are too close to each other $(<0.3 \AA)$, were deleted. The average grain sizes of the ng-diamond model samples ranges from 2 to $25 \mathrm{~nm}$. The constructed models were then relaxed at $300 \mathrm{~K}$ and had an ambient pressure with a relaxation time of 200 ps. The GB thickness (GBT) of relaxed 3D ng-diamond samples was calculated with a common neighbor analysis method. ${ }^{30}$ GBT decreases with increasing grain size, ranging from 0.81 (grain size $\sim 2 \mathrm{~nm}$ ) to $0.72 \mathrm{~nm}$ (grain size $\sim 25 \mathrm{~nm}$ ). This scale is similar to that for nanocrystalline metals. ${ }^{31}$ The bond-length distributions of diamond nanograins are shown in Supplementary Fig. 19. The bond lengths at the GBs are clearly in the range of bonding, indicating stable and equilibrated grain boundaries in our ng-diamond model. The 3D nt-diamond samples with sub-grain nanotwins were then built on the basis of ng-diamond samples (i.e., ntdiamond samples had grain size and orientation identical to those of the corresponding ng-diamond; see Supplementary Fig. 1). The largest sample contained about 30 million carbon atoms.

The MD simulations were then performed on these samples using the popular LAMMPS code. ${ }^{32}$ Atomic configurations were visualized and analyzed with the OVITO package. ${ }^{33}$ Periodic boundary conditions were applied to account for the confinement effects in large bulk materials. Interactions of $\mathrm{C}-\mathrm{C}$ bonds were described by both Tersoff potential ${ }^{19}$ and Albe analytical potential, ${ }^{21}$ which properly describes the carbon $s p^{3}$ bonding and has been successfully used in many ng-diamond simulations. ${ }^{34}$ For the ratios of intrinsic stacking fault energy $\left(\gamma_{\text {isf }}\right)$, unstable twinning fault energy $\left(\gamma_{\mathrm{utf}}\right)$, and unstable twinning migration energy $\left(\gamma_{\mathrm{utm}}\right)$ to unstable stacking fault energy $\left(\gamma_{\text {usf }}\right)$, which are the deciding factors for plastic deformation modes, Tersoff and Albe potentials give results consistent with those from first-principles calculations (Supplementary Table 1), indicating both potentials can capture the essence the plastic deformation properly. The model samples after structure optimization were compressed with either a displacement-controlled uniaxial compression mode or a force-controlled one. The isothermal-isobaric (NPT) scheme was used in our simulations for both potentials, with a timestep of $0.001 \mathrm{ps}$ and a relaxation time $200 \mathrm{ps}$ at $300 \mathrm{~K}$ and ambient pressure. For displacement-controlled uniaxial compression, a constant strain rate of $5 \times 10^{8} \mathrm{~s}^{-1}$ were applied on the model samples and the compressive strength was set as the peak stress of the corresponding stress-strain curve. For force-controlled compression, a constant uniaxial compressive stress was applied to the sample in a stepwise manner. At each step, a constant stress was applied with a duration of $300 \mathrm{ps}$ and the sample was allowed to relax in the directions perpendicular to the load. The deformation curve (strain as a function of stress dwelling time) was recorded and the compressive strength was determined as the critical stress under which plastic strain and strain rate increased abruptly.

\section{First-Principles Calculation}

The GSFE curves were calculated within the framework of electronic density functional theory, as implemented in the Vienne $\mathrm{Ab}$ initio Simulation Package. ${ }^{35}$ The exchange and correlation interactions were described in the local density approximation (LDA-CAPZ). ${ }^{36}$ Interactions between ions and valence electrons were modeled by the projectoraugmented wave method. ${ }^{37}$ The kinetic cutoff energy for plane waves was $600 \mathrm{eV}$ for diamond and $\mathrm{CBN}$, and $500 \mathrm{eV}$ for $\mathrm{Al}, \mathrm{Cu}$, and Ni. The k-point separation in Brillouin zone was $5 \times 7 \times 1$ for all orthorhombic lattices. To calculate GSFE curves of on closed-packed (111) surface along the $[11 \overline{2}]$ and $[1 \overline{1} 0]$ directions, an orthorhombic lattice was used, which included a slab consisting of 12 atomic layers along the [111] direction. Crystallographic axes $\mathbf{a}, \mathbf{b}$, and $\mathbf{c}$ for the orthorhombic lattice were parallel to the $[1 \overline{1} 0],[11 \overline{2}]$, and $[111]$ directions of the diamond, $\mathrm{CBN}, \mathrm{Al}, \mathrm{Cu}$, and Ni cubic lattice, respectively. To avoid interaction between two adjacent slabs, a vacuum space of $15 \AA$ was added to the top and bottom of the slab along the [111] direction. The slab was separated into two halves along slip plane and the two halves of slab were displaced relative to each other along slip directions. The GSFE curve can be obtained by plotting the excess energy with the displaced distance.

\section{DATA AVAILABILITY}

The authors declare that the data supporting the findings of this study are available within the paper and its Supplementary Information files.
Received: 2 July 2019; Accepted: 13 November 2019; Published online: 05 December 2019

\section{REFERENCES}

1. Meyers, M. A., Mishra, A. \& Benson, D. J. Mechanical properties of nanocrystalline materials. Prog. Mater. Sci. 51, 427-556 (2006).

2. Hall, E. The deformation and ageing of mild steel: III discussion of results. Proc. Phys. Soc. Sect. B. 64, 747-753 (1951).

3. Petch, N. J. The cleavage strength of polycrystals. J. Iron Steel Inst. 174, 25-28 (1953).

4. Hu, J., Shi, Y. N., Sauvage, X. \& Lu, K. Grain boundary stability governs hardening and softening in extremely fine nanograined metals. Science 355, 1292-1296 (2017).

5. Lu, K. Stabilizing nanostructures in metals using grain and twin boundary architectures. Nat. Rev. Mater. 1, 1-13 (2016).

6. Wang, J. et al. Detwinning mechanisms for growth twins in face-centered cubic metals. Acta Mater. 58, 2262-2270 (2010).

7. Schiøtz, J., DiTolla, F. D. \& Jacobsen, K. W. Softening of nanocrystalline metals at very small grain sizes. Nature 391, 561-563 (1998).

8. Swygenhoven, H. V. Grain boundaries and dislocations. Science 296, 66-67 (2002).

9. Lu, L., Chen, X., Huang, X. \& Lu, K. Revealing the maximum strength in nanotwinned copper. Science 323, 607-610 (2009).

10. Li, X., Wei, Y., Lu, L., Lu, K. \& Gao, H. Dislocation nucleation governed softening and maximum strength in nano-twinned metals. Nature 464, 877-880 (2010).

11. Schiotz, J., Vegge, T., Di Tolla, F. D. \& Jacobsen, W. Atomic-scale simulations of the mechanical deformation of nanocrystalline metals. Phys. Rev. B. 60, 11971 (1999).

12. Schiøtz, J. \& Jacobsen, K. W. A maximum in the strength of nanocrystalline copper. Science 301, 1357-1359 (2003).

13. Dubrovinskaia, N. et al. Superhard nanocomposite of dense polymorphs of boron nitride: noncarbon material has reached diamond hardness. Appl. Phys. Lett. 90, 101912 (2007)

14. Sumiya, H. \& Irifune, T. Hardness and deformation microstructures of nanopolycrystalline diamonds synthesized from various carbons under high pressure and high temperature. J. Mater. Res. 22, 2345-2351 (2007).

15. Tian, Y. et al. Ultrahard nanotwinned cubic boron nitride. Nature 493, 385-388 (2013).

16. Huang, Q. et al. Nanotwinned diamond with unprecedented hardness and stability. Nature 510, 250-253 (2014).

17. Mousavi, S. M. T., Zou, G., Zhou, H. \& Gao, H. Anisotropy governs strain stiffening in nanotwinned-materials. Nat. Commun. 9, 1586 (2018).

18. Li, B., Sun, H. \& Chen, C. Reply to 'Anisotropy governs strain stiffening in nanotwinned-materials'. Nat. Commun. 9, 1585 (2018).

19. Tersoff, J. Modeling solid-state chemistry: Interatomic potentials for multicomponent systems. Phys. Rev. B. 39, 5566-5568 (1989).

20. Tabor, D. The Hardness of Metals (The Clarendon Press, 1951).

21. Erhart, P. \& Albe, K. Analytical potential for atomistic simulations of silicon, carbon, and silicon carbide. Phys. Rev. B. 71, 035211 (2005).

22. Frøseth, A., Van Swygenhoven, H. \& Derlet, P. M. The influence of twins on the mechanical properties of nc-Al. Acta Mater. 52, 2259-2268 (2004).

23. Van Swygenhoven, H., Spaczer, M., Caro, A. \& Farkas, D. Competing plastic deformation mechanisms in nanophase metals. Phys. Rev. B. 60, 22 (1999).

24. Yip, S. The strongest size. Nature 391, 532-533 (1998).

25. Zhang, X., Romanov, A. E. \& Aifantis, E. C. A simple physically based phenomenological model for the strengthening/softening behavior of nanotwinned copper. J. Appl. Mech. 82, 121005 (2015).

26. Xiao, J. et al. Dislocation behaviors in nanotwinned diamond. Sci. Adv. 4, eaat8195 (2018).

27. Xiao, J. et al. Strengthening-softening transition in yield strength of nanotwinned Cu. Scr. Mater. 162, 372-376 (2019).

28. Taheri Mousavi, S. M., Zhou, H., Zou, G. \& Gao, H. Transition from source- to stresscontrolled plasticity in nanotwinned materials below a softening temperature. npj Comput. Mater. 5, 2 (2019).

29. Voronoi, G. Nouvelles applications des parametres continus a la theorie des formes quadratiques. J. fur die Reine und Angew. Mathematik 134, 198-287 (1908).

30. Honeycutt, J. D. \& Andersen, H. C. Modecular dynamics study of melting and freezing of small Lennard-Jones cluseters. J. Phys. Chem. 91, 4950-4963 (1987).

31. Shimokawa, T., Nakatani, A. \& Kitagawa, H. Grain-size dependence of the relationship between intergranular and intragranular deformation of nanocrystalline Al by molecular dynamics simulations. Phys. Rev. B. 71, 224110 (2005). 
32. Phimpton, S. Fast parallel algorithms for short-range molecular dynamics. J. Comput. Phys. 117, 1-19 (1995).

33. Stukowski, A. Visualization and analysis of atomistic simulation data with OVITO-the Open Visualization Tool. Model. Simul. Mater. Sci. Eng. 18, 015012 (2010).

34. Remediakis, I. N., Kopidakis, G. \& Kelires, P. C. Softening of ultra-nanocrystalline diamond at low grain sizes. Acta Mater. 56, 5340-5344 (2008).

35. Kresse, G. \& Furthmüller, J. Efficient iterative schemes for ab initio total-energy calculations using a plane-wave basis set. Phys. Rev. B. 54, 11169 (1996).

36. Perdew, J. P. \& Zunger, A. Self-interaction correction to density-functional approximations for many-electron systems. Phys. Rev. B. 23, 5048 (1981).

37. Kresse, G. \& Joubert, D. From ultrasoft pseudopotentials to the projector augmented-wave method. Phys. Rev. B 59, 1758 (1999).

\section{ACKNOWLEDGEMENTS}

This work was supported by the National Key R\&D Program of China (2018YFA0703400) and the National Natural Science Foundation of China (NSFC, Grant Numbers 51925105, 51525205 and 51771165).

\section{AUTHOR CONTRIBUTIONS}

Y.J.T. conceived the project. B.W. and B.X. performed the calculations. B.W., B.X., Y.B. W., and Y.J.T. analyzed the data and co-wrote the paper. All authors discussed the results and commented on the manuscript.

\section{COMPETING INTERESTS}

The authors declare no competing interests.

\section{ADDITIONAL INFORMATION}

Supplementary information is available for this paper at https://doi.org/10.1038/ s41524-019-0256-2.

Correspondence and requests for materials should be addressed to Y.T.

Reprints and permission information is available at http://www.nature.com/ reprints

Publisher's note Springer Nature remains neutral with regard to jurisdictional claims in published maps and institutional affiliations.

Open Access This article is licensed under a Creative Commons Attribution 4.0 International License, which permits use, sharing, adaptation, distribution and reproduction in any medium or format, as long as you give appropriate credit to the original author(s) and the source, provide a link to the Creative Commons license, and indicate if changes were made. The images or other third party material in this article are included in the article's Creative Commons license, unless indicated otherwise in a credit line to the material. If material is not included in the article's Creative Commons license and your intended use is not permitted by statutory regulation or exceeds the permitted use, you will need to obtain permission directly from the copyright holder. To view a copy of this license, visit http://creativecommons. org/licenses/by/4.0/.

(c) The Author(s) 2019 